\title{
MATHEMATICAL ASPECTS OF THE MECHANICS OF LEFT VENTRICULAR CONTRACTION
}

\author{
R.M. SHOUCRI \\ Department of Mathematics and Computer Science, Royal Military College of Canada, Kingston, Ontario, Canada.
}

\begin{abstract}
Large elastic deformation is used in order to model the mechanics of left ventricular contraction. The active force generated by the myocardium is modeled as force/unit myocardial volume in the mathematical formalism describing the local equilibrium of forces in the myocardium. Expressions for the stress components are derived by assuming a cylindrical geometry for the left ventricle, the total stress is expressed as the sum of a component due to the deformation of the passive medium of the myocardium and an active component induced by the tension in the muscular fibers. It is shown that knowledge of the tension generated by the muscular fiber in the myocardium can lead to useful information for the estimation of the pseudo-strain energy function used to express the stress-strain constitutive relations in a non-linear model.
\end{abstract}

Keywords: active force of the myocardium, cardiac mechanics, mathematical modeling of ventricular contraction, pressure-volume relation in the left ventricle.

\section{INTRODUCTION}

Many articles have been devoted to the study of stress-strain relations in the myocardium and how to formulate a pseudo-strain energy function $W$ that is used to derive the constitutive relations between stress and strain [1-4]. Previous studies by the authors [5-9] have shown how the active force developed by the myocardium can be modeled as force generated by unit volume of the myocardium in the mathematical formalism used to describe the local equilibrium of forces. This mathematical approach was successfully developed by using large elastic deformation $[5,8]$ as well as linear elasticity [6]. For this purpose the total stress in the myocardium is expressed as the sum of a contribution due to the stress generated by the deformation of the passive isotropic medium of the myocardium, and a contribution coming from the stress induced by the active muscular fibers. A mathematical description of how this splitting is done can be found in the work of Spencer [10]. The approach used in this study is similar to the approach used in some studies in which the pseudostrain energy function $W$ is split into the sum of an isotropic component and an anisotropic component in the form $W=W_{\text {iso }}+W_{\text {aniso }}$ [11], the link between the two approaches is evident from what follows. We also show in this study how the calculation of the stress in the myocardium can be based on the knowledge of the muscular fiber tension $T$ without an explicit knowledge of the pseudo-strain energy function $W$, which presents a possible way to estimate $W$ as a result of the method of calculation shown in this study.

\section{ACTIVE FORCE OF THE MYOCARDIUM}

The myocardium is represented as a thick-walled cylinder (see Fig. $1 \&$ Fig. 2), with the myocardial muscular fibers arranged in a helical way in a passive soft incompressible medium assumed to be isotropic. Inertia forces and viscous forces are neglected in the quasi-static approximation used in this study. The unit tangent vector $\tau$ in the direction of the helical fiber makes an angle $\gamma(r)$ with the unit vector $\boldsymbol{e}_{\theta}$ pointing in the circumferential direction and can be expressed in the form (see Fig. 1)

$$
\boldsymbol{\tau}=\boldsymbol{e}_{\theta} \cos \gamma(r)+\boldsymbol{e}_{z} \sin \gamma(r)
$$

(C) 2010 WIT Press, www.witpress.com

ISSN: 1755-7437 (paper format), ISSN: 1755-7445 (online), http://journals.witpress.com

DOI: 10.2495/DNE-V5-N2-173-188 


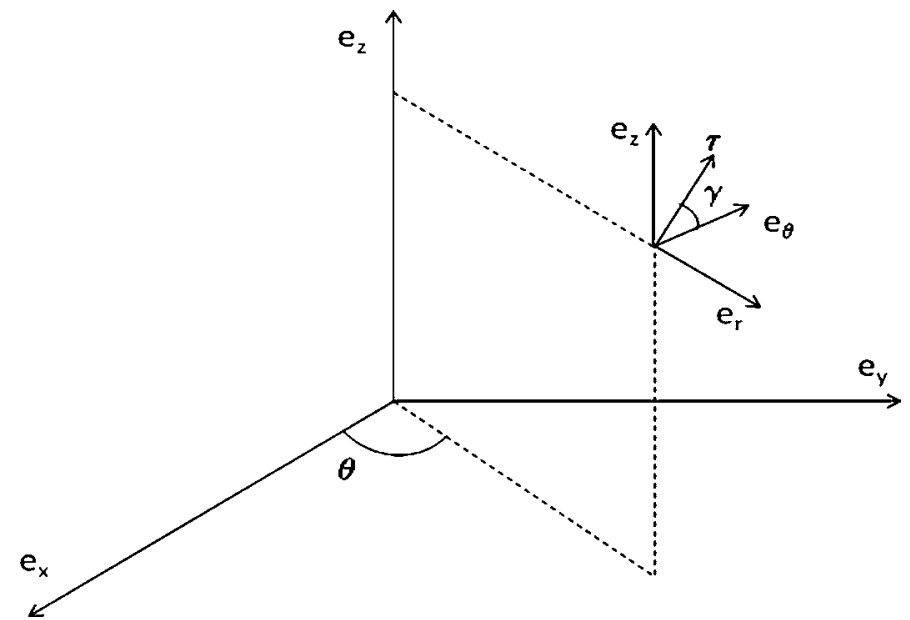

Figure 1: $\left(\boldsymbol{e}_{\boldsymbol{r}}, \boldsymbol{e}_{\theta}, \boldsymbol{e}_{z}\right)$ are three unit vectors in a cylindrical coordinates system. A unit vector $\boldsymbol{\tau}$ tangent to the direction of a helical muscular fiber in the myocardium makes an angle $\gamma(r)$ with the unit vector $\boldsymbol{e}_{\boldsymbol{\theta}}$ in the circumferential direction.

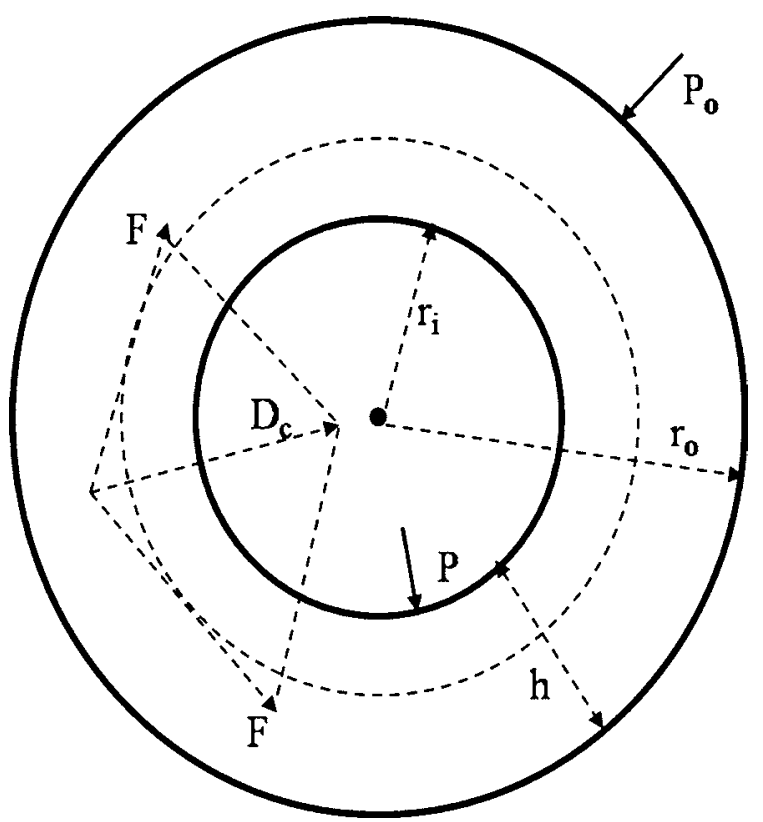

Figure 2: The left ventricle is represented as a thick-walled cylinder, with inner radius $r_{i}$ and outer radius $r_{o}$, wall thickness $h=r_{o}-r_{i}$. A helical fiber is projected as a dotted circle on the cross-section. The radial active force/unit volume of the myocardium is $D_{c}(r)=-D_{r}(r)$ in the notation of the text. $P_{i}$ is the left ventricular pressure, $P_{o}$ is the outer pressure on the epicardium. 
Notice in this case div $\tau=0$ which in conjunction with the result given by Peskin [12] allows one to express the active force/unit myocardial volume in the form

$$
\boldsymbol{D}=\left(D_{r}, D_{\theta}, D_{z}\right)=\frac{\mathrm{d}(T \tau)}{\mathrm{d} s}
$$

where $T(r, z)$ is the tension/unit myocardial area generated by the muscular fibers, $\mathrm{d} / \mathrm{d} s=\tau \cdot \nabla$ with $\nabla$ standing for the gradient symbol which in cylindrical coordinates is given by

$$
\nabla=\boldsymbol{e}_{r} \frac{\partial}{\partial r}+\boldsymbol{e}_{\theta} \frac{1}{r} \frac{\partial}{\partial \theta}+\boldsymbol{e}_{z} \frac{\partial}{\partial z}
$$

The unit vectors in cylindrical coordinates are given by $\boldsymbol{e}_{r}, \boldsymbol{e}_{\boldsymbol{\theta}}$ and $\boldsymbol{e}_{z}$. Performing the differentiation on the right hand side of eqn (2) gives the following results

$$
\begin{gathered}
D_{r}=-\frac{(\cos \gamma(r))^{2}}{r} T \\
D_{e}=\sin \gamma(r) \cos \gamma(r) \frac{\partial T}{\partial z} \\
D_{z}=(\sin \gamma(r))^{2} \frac{\partial T}{\partial z}
\end{gathered}
$$

The active stress tensor $\boldsymbol{q}$ (components $q_{i j}$ ) represents the stress components in an orthogonal cylindrical coordinate system induced by the muscular fiber stress $T(r, z)$. By taking into consideration eqn (1), the components of the active stress $q_{i j}=T \tau_{i j}$ (see [13]) are given in a cylindrical coordinate system by the following relations:

$$
\begin{aligned}
& q_{\theta \theta}=T(\cos \gamma(r))^{2} \quad q_{\mathrm{ZZ}}=T(\sin \gamma(r))^{2} \\
& q_{\theta z}=q_{z \theta}=T(\sin \gamma(r))(\cos \gamma(r)) \quad q_{r \theta}=q_{r z}=q_{r r}=0
\end{aligned}
$$

The relation between the active force/unit myocardial volume $\boldsymbol{D}=\left(D_{r}, D_{\theta}, D_{z}\right)$ and the active stress tensor $\boldsymbol{q}$ can be obtained by using Gauss theorem. The traction force $\boldsymbol{F}_{\boldsymbol{a}}$ acting on a closed surface $A$ enclosing a volume $V$ inside the myocardium can be expressed in the form

$$
\boldsymbol{F}_{a}=\iint \boldsymbol{q} \mathrm{d} \boldsymbol{A}=\iiint(\operatorname{div} \boldsymbol{q}) \mathrm{d} V
$$

Consequently the active force/unit myocardial volume can be expressed as

$$
\boldsymbol{D}=\operatorname{div} \boldsymbol{q}
$$

We take into consideration eqn (5) and the fact that the solution is independent of the $\theta$-coordinate (symmetry around the $z$-axis). The expression of the divergence in cylindrical coordinates (see [8]) gives

$$
\left(D_{r}, D_{\theta}, D_{z}\right)=-\boldsymbol{e}_{r} \frac{(\cos \gamma)^{2}}{r} T+\boldsymbol{e}_{\theta}(\sin \gamma)(\cos \gamma) \frac{\partial T}{\partial z}+\boldsymbol{e}_{z}(\sin \gamma)^{2} \frac{\partial T}{\partial z}
$$


from which we retrieve the relations given in eqn (4). We notice from eqns (4) and (5) that we have the relations

$$
D_{r}=-\frac{q_{\theta \theta}}{r} \quad D_{\theta}=\frac{\partial q_{z \theta}}{\partial z} \quad D_{z}=\frac{\partial q_{z z}}{\partial z}
$$

\section{QUASI-STATIC EQUILIBRIUM CONDITION}

We assume symmetry around the $z$-axis (solution independent of $\theta$ ). Under this assumption the total stress components $t_{i j}$ must satisfy the quasi-static equilibrium condition $\operatorname{div} \boldsymbol{t}=0$, which in cylindrical coordinates is expressed as follows:

$$
\begin{gathered}
\frac{\partial t_{r r}}{\partial r}+\frac{t_{r r}-t_{\theta \theta}}{r}+\frac{t_{z r}}{\partial z}=0 \\
\frac{1}{r^{2}} \frac{\partial\left(r^{2} t_{r \theta}\right)}{\partial r}+\frac{\partial t_{z \theta}}{\partial z}=0 \\
\frac{1}{r} \frac{\partial\left(r t_{r z}\right)}{\partial r}+\frac{\partial t_{z z}}{\partial z}=0
\end{gathered}
$$

We shall simplify the problem by assuming that $t_{z r}$ and $t_{z \theta}$ are independent of $z$ variable, which gives

$$
r^{2} t_{r \theta}=\text { const }=H_{1} \quad r t_{r z}=\text { const }=H_{2}
$$

The boundary conditions for the radial stress on the surface of the cylinder can be expressed as follows:

$$
t_{r r}\left(r_{i}\right)=-P_{i} \quad t_{r r}\left(r_{o}\right)=-P_{o}
$$

We now use the decomposition $t_{i j}=\sigma_{i j}+q_{i j}$. By using eqns (9), eqns (10) can be written in the form

$$
\begin{gathered}
\frac{\partial \sigma_{r r}}{\partial r}+\frac{\sigma_{r r}-\sigma_{\theta \theta}}{r}+\frac{\partial}{\partial z}\left(\sigma_{r r}+\int_{z} D_{r} d z\right)=0 \\
\frac{1}{r^{2}} \frac{\partial\left(r^{2} \sigma_{r \theta}\right)}{\partial r}+\frac{\partial}{\partial z}\left(\sigma_{z \theta}+\int_{z} D_{\theta} d z\right)=0 \\
\frac{1}{r} \frac{\partial\left(r \sigma_{r z}\right)}{\partial r}+\frac{\partial}{\partial z}\left(\sigma_{z z}+\int_{z} D_{z} d z\right)=0
\end{gathered}
$$

The force/unit volume of the myocardium $D_{r}$ appearing in eqn (13a) is similar, but not identical, to the introduction of the derivative of a hydrostatic pressure in eqn (26) of reference [14]. Another, but different, attempt to divide the stress in the myocardium into two components can be found in [15].

Some simplifying assumptions concerning the dependence on the $z$ variable will be introduced. By noting that $t_{r \theta}=\sigma_{r \theta}\left(q_{r \theta}=0\right), t_{r z}=\sigma_{r z}\left(q_{r z}=0\right)$ and $t_{r r}=\sigma_{r r}\left(q_{r r}=0\right)$, one can write by referring to eqns (11) and (12)

$$
\begin{array}{cc}
r^{2} \sigma_{r \theta}=\text { const }=H_{1} & r \sigma_{r z}=\text { const }=H_{2} \\
\sigma_{r r}\left(r_{i}\right)=-P_{i} & \sigma_{r r}\left(r_{o}\right)=-P_{o}
\end{array}
$$

Two useful relations that we shall apply in our calculations are: first the moment of forces $M$ around the $z$-axis is assumed zero (no external moment applied around the $z$-axis). 


$$
M=2 \pi \int_{r i}^{r o}\left(\sigma_{z \theta}+q_{z \theta}\right) r^{2} d r=0
$$

from which we can assume that

$$
\sigma_{z \theta}=-q_{z \theta}
$$

Second from the equilibrium of forces in the vertical direction we get

$$
\sigma_{z z}+q_{z z}+\xi=0
$$

where $\xi$ is the traction on the cross-section and it is assumed to vary linearly over the cross-section

$$
\xi=\xi_{\text {end }}\left(\frac{r_{o}-r}{r_{o}-r_{1}}\right)+\xi_{\text {epi }}\left(\frac{r-r_{i}}{r_{o}-r_{1}}\right)
$$

We have taken in our calculation the stress at the endocardium $\xi_{\text {end }}=0.5 P_{i}$ and at the epicardium $\xi_{\text {epi }}=-0.01 P_{i}$ based on estimate of the average traction $\xi_{a v}=\left(P_{i} r_{i}^{2}-P_{o} r_{o}^{2}\right) /\left(r_{o}^{2}-r_{i}^{2}\right)$.

\section{DEFORMATION GRADIENT}

The thick-walled cylindrical structure representing the left ventricle is supposed to have a stress free configuration with coordinates $(R, \Theta, Z)$, at end-diastole the cylindrical coordinates are represented by $\left(r_{e d}, \theta_{e d}, z_{e d}\right)$ and finally by $(r, \theta, z)$ during the systolic phase. The coordinate transformation from one configuration to the other is expressed as follows:

$$
\begin{gathered}
r_{e d}=r_{e d}(R) \quad \theta_{e d}=\alpha_{1} \Theta \quad z_{e d}=k_{1} z \\
r=r\left(r_{e d}\right) \quad \theta=a_{2} \theta_{e d}+\psi_{2} z_{e d}+\chi\left(r_{e d}\right) \quad z=k_{2 z} z_{e d}+k_{2 \theta} \theta_{e d}+\omega\left(r_{e d}\right)
\end{gathered}
$$

The two preceding equations can be combined together to give

$$
r=r(R) \quad \theta=a \Theta+\psi Z+\chi(R) \quad z=k_{z} Z+k_{\theta} \Theta+\omega(R)
$$

The deformation gradient $F_{1}$ for the transformation $(R, \Theta, Z) \rightarrow\left(r_{e d}, \theta_{e d}, z_{e d}\right)$ can be expressed as follows (see [2]):

$$
F_{1}=\left[\begin{array}{ccc}
\frac{\partial r_{e d}}{\partial R} & \frac{1}{R} \frac{\partial r_{e d}}{\partial \Theta} & \frac{\partial r_{e d}}{\partial Z} \\
r_{e d} \frac{\partial \theta_{e d}}{\partial R} & \frac{r_{e d}}{R} \frac{\partial \theta_{e d}}{\partial \Theta} & r_{e d} \frac{\partial \theta_{e d}}{\partial Z} \\
\frac{\partial z_{e d}}{\partial R} & \frac{1}{R} \frac{\partial z_{e d}}{\partial \Theta} & \frac{\partial z_{e d}}{\partial Z}
\end{array}\right]=\left[\begin{array}{ccc}
\frac{\mathrm{d} r_{e d}}{\mathrm{~d} R} & 0 & 0 \\
0 & \alpha_{1} \frac{r_{e d}}{R} & 0 \\
0 & 0 & k_{1}
\end{array}\right]
$$

The deformation gradient $F_{2}$ for the transformation $\left(r_{e d}, \theta_{e d}, z_{e d}\right) \rightarrow(r, \theta, z)$ is given by

$$
F_{2}=\left[\begin{array}{cccc}
\frac{\partial r}{\partial r_{e d}} & \frac{1}{r_{e d}} \frac{\partial r}{\partial \theta_{e d}} & \frac{\partial r}{\partial z_{e d}} \\
r \frac{\partial \theta}{\partial \theta_{e d}} & \frac{r}{r_{e d}} \frac{\partial \theta}{\partial \theta_{e d}} & r \frac{\partial \theta}{\partial z_{e d}} \\
\frac{\partial z}{\partial r_{e d}} & \frac{1}{r_{e d}} \frac{\partial z}{\partial \theta_{e d}} & \frac{\partial z}{\partial z_{e d}}
\end{array}\right]=\left[\begin{array}{ccc}
\frac{\mathrm{d} r}{\mathrm{~d} r_{e d}} & 0 & 0 \\
r \frac{\mathrm{d} \chi}{\mathrm{d} r_{e d}} & \alpha_{2} \frac{r}{r_{e d}} & r \psi_{2} \\
\frac{\mathrm{d} \omega}{\mathrm{d} r_{e d}} & \frac{k_{2 \theta}}{r_{e d}} & k_{2 z}
\end{array}\right]
$$


The deformation gradient $F=F_{2} * F_{1}$ of the combined transformation is given by

$$
F=\left[\begin{array}{ccc}
\frac{\partial r}{\partial R} & \frac{1}{R} \frac{\partial r}{\partial \Theta} & \frac{\partial r}{\partial Z} \\
r \frac{\partial \theta}{\partial R} & \frac{r}{R} \frac{\partial \theta}{\partial \Theta} & r \frac{\partial \theta}{\partial Z} \\
\frac{\partial z}{\partial R} & \frac{r}{R} \frac{\partial z}{\partial \Theta} & \frac{\partial z}{\partial Z}
\end{array}\right]=\left[\begin{array}{ccc}
\frac{\mathrm{d} r}{d R} & 0 & 0 \\
r \frac{\mathrm{d} \chi}{d R} & a \frac{r}{R} & r \psi \\
\frac{\mathrm{d} \omega}{\mathrm{d} R} & \frac{k_{\theta}}{R} & k_{z}
\end{array}\right]
$$

By assuming that the contraction takes place at constant volume, the incompressibility constraint is expressed as follows:

$$
I_{3}=(\operatorname{det} F)^{2}=\left(\operatorname{det} F_{2}\right)^{2} *\left(\operatorname{det} F_{1}\right)^{2}=1
$$

where $I_{3}$ is the third invariant of the matrix $F$. By calculating the determinants in eqn (26), equating to one and then integrating we get

$$
\begin{aligned}
\frac{\mathrm{d} r}{\mathrm{~d} R}=\frac{R}{K r}, & K r^{2}-R^{2}=K r_{i}{ }^{2}-R_{i}^{2} \\
\frac{\mathrm{d} r_{e d}}{\mathrm{~d} R}=\frac{R}{K_{1} r_{e d}}, & K_{1} r_{e d}^{2}-R^{2}=K_{1} r_{i e d}^{2}-R_{i}^{2} \\
\frac{\mathrm{d} r}{\mathrm{~d} r_{e d}}=\frac{r_{e d}}{K_{2} r}, & K_{2} r^{2}-r_{e d}^{2}=K_{2} r_{i}^{2}-r_{\text {ied }}^{2}
\end{aligned}
$$

The constants in eqns (27)-(29) are defined as follows: $K_{1}=\alpha_{1} k_{1}, K_{2}=\alpha_{2} k_{2 z}-\psi_{2} k_{2 \theta}$ and $K=K_{1} K_{2}=$ $\alpha_{1} k_{1} \alpha_{2} k_{2 z}-\alpha_{1} k_{2 \theta} k_{1} \psi_{2}=\alpha k_{z}-k_{\theta} \psi$. The inner radius is $R_{i}$ in the stress free configuration and $r_{i}$ during the systolic phase.

The helical configuration of a muscular fiber in the myocardium is supposed to be confined to a cylindrical surface. A unit vector $\tau$ in the direction of the fiber in the deformed configuration will make an angle $\gamma(r)$ calculated with respect to the circumferential direction (see Fig. 1), in the stress free configuration the corresponding unit vector is $\tau_{o}$ and the angle is $\gamma_{o}(r)$, the relation between these two vectors are given by

$$
\tau=\frac{1}{\lambda_{f}} F \tau_{o}
$$

where $\lambda_{f}$ is the stretch ratio in the direction of the fiber. By using eqn (25) we can write

$$
\begin{gathered}
\cos (\gamma(r))=\frac{1}{\lambda_{f}}\left[\frac{a r}{R} \cos \left(\gamma_{o}(r)\right)+(r \psi) \sin \left(\gamma_{o}(r)\right)\right] \\
\sin (\gamma(r))=\frac{1}{\lambda_{f}}\left[\frac{k_{\theta}}{R} \cos \left(\gamma_{o}(r)\right)+k_{z} \sin \left(\gamma_{o}(r)\right)\right]
\end{gathered}
$$

We can model first $\gamma_{o}(r)$ and then use eqns $(31,32)$ to calculate $\gamma(r)$. But in view of the method of calculation we are going to use later on, it is easier to model directly $\gamma(r)$ and then to calculate back $\gamma_{o}(r)$ at the end if needed. Consequently we shall assume directly either a linear variation for $\gamma(r)$ across the myocardium of the form 


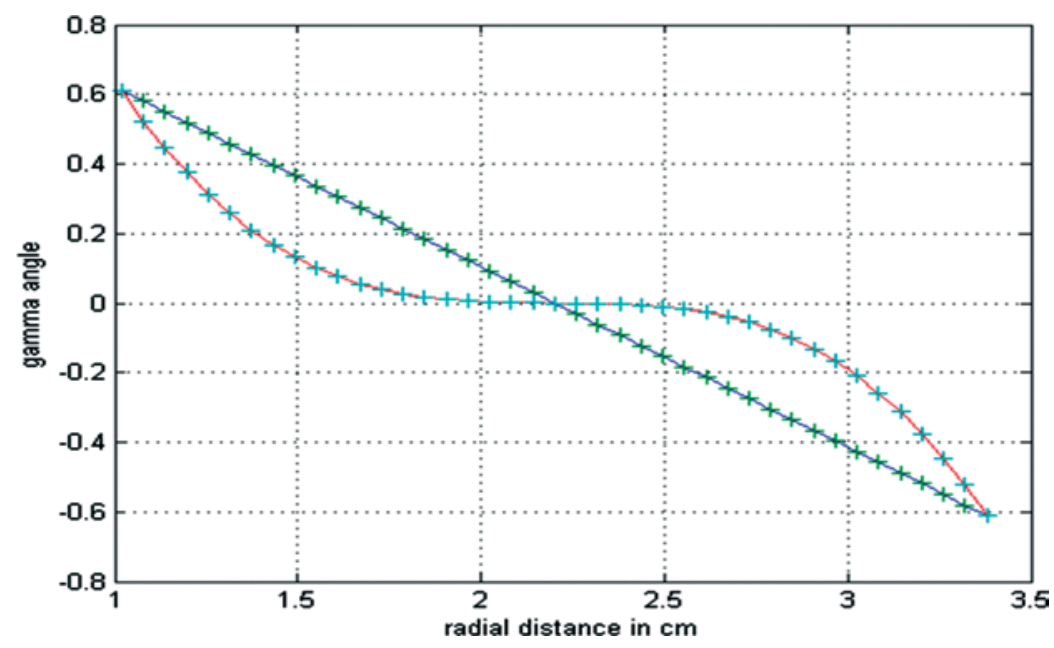

Figure 3: Linear model (eqn (33)) and non-linear model (eqn (34)) for the variation of $\gamma(r)$ with the radial distance $r$ from endocardium to epicardium.

$$
\gamma(r)=\gamma_{\text {end }}\left(\frac{r_{o}-r}{r_{o}-r_{i}}\right)+\gamma_{\text {epi }}\left(\frac{r-r_{i}}{r_{o}-r_{i}}\right)
$$

or a cubic variation of the form [16]

$$
\gamma(r)=\gamma_{\text {end }}\left(\frac{r_{o}+r_{i}-2 r}{r_{o}-r_{i}}\right)^{3}
$$

In our calculation, we have taken $\gamma_{\text {end }}=35^{\circ}$ and $\gamma_{e p i}=-35^{\circ}$. The radial variation for these two models is shown in Fig. 3. $\lambda_{f}$ can be calculated from eqns (31) and (32) by eliminating $\cos \left(\gamma_{o}\right)$ and $\sin \left(\gamma_{o}\right)$ from these two equations.

\section{CONSTITUTIVE RELATIONS}

The relation between stress and strain is expressed with what is called constitutive relation. It is expressed by calculating the left or right Cauchy-Green deformation tensors, respectively, $B=F^{*} F^{T}$ or $C=F^{T *} F$. In this study we shall use the model of Humphrey and Yin [1] which focus on a subclass of transverse isotropic material with pseudo-strain energy function $W$ given by

$$
W=W\left(I_{1}, \lambda_{f}\right),
$$

where $I_{1}$ is the first invariant of the matrix $B, I_{1}=\operatorname{tr}(B)$. Written explicitly the expression of $B$ becomes

$$
B=\left[\begin{array}{ccc}
\left(\frac{\mathrm{d} r}{\mathrm{~d} R}\right)^{2} & r \frac{\mathrm{dr}}{\mathrm{d} R} \frac{\mathrm{d} \chi}{\mathrm{d} R} & \frac{\mathrm{d} r}{\mathrm{~d} R} \frac{\mathrm{d} \omega}{\mathrm{d} R} \\
r \frac{\mathrm{d} r}{\mathrm{~d} R} \frac{\mathrm{d} \chi}{\mathrm{d} R} & \left(r \frac{\mathrm{d} \chi}{\mathrm{d} R}\right)^{2}+\left(\frac{a r}{R}\right)^{2}+(r \psi)^{2} & r \frac{\mathrm{d} \chi}{\mathrm{d} R} \frac{\mathrm{d} \omega}{\mathrm{d} R}+\frac{a r k_{\theta}}{R^{2}}+r \psi k_{z} \\
\frac{\mathrm{d} r}{\mathrm{~d} R} \frac{\mathrm{d} \omega}{\mathrm{d} R} & r \frac{\mathrm{d} \chi}{\mathrm{d} R} \frac{\mathrm{d} \omega}{\mathrm{d} R}+\frac{a r k_{\theta}}{R^{2}}+r \psi k_{z} & \left(\frac{\mathrm{d} \omega}{\mathrm{d} R}\right)^{2}+\frac{k_{\theta}^{2}}{R^{2}}+k_{z}^{2}
\end{array}\right]
$$


By using eqn (36) the Cauchy stress tensor $t$ can be expressed as follows (see [1]):

$$
t_{i j}=-p \delta_{i j}+2 W_{I 1} B_{i j}+W_{\lambda f} \lambda_{f} \tau_{i} \tau_{j}
$$

where $W_{\mathrm{I} 1}=\partial W / \partial I_{1}$ and $W_{\lambda f}=\partial W / \partial \lambda, p$ is a Lagrange multiplier introduced to express the incompressibility condition that the contraction of the myocardium is assumed to take place at constant volume. By writing the total stress $t_{i j}=\sigma_{i j}+q_{i j}$ it is possible to split eqn (37) into two parts. The first part has the form

$$
\sigma_{i j}=-p \delta_{i j}+2 W_{I 1} B_{i j}
$$

where $\sigma_{i j}$ is the stress resulting from the deformation of the isotropic passive medium of the myocardium, and the second part has the form

$$
q_{i j}=W_{\lambda f} \lambda_{f} \tau_{i} \tau_{j}, \quad \text { with } T=W_{\lambda f} \lambda_{f}
$$

where $q_{i j}$ is the stress induced by the muscular fiber tension $T(r, z)$ and reflects the directional character of the stress. Equation (38) can be written in explicit form as follows:

$$
\begin{gathered}
\sigma_{r \theta}=2 W_{I 1} r \frac{\mathrm{d} r}{\mathrm{~d} R} \frac{\mathrm{d} \chi}{\mathrm{d} R}=\frac{H_{1}}{r^{2}} \\
\sigma_{r z}=2 W_{l 1} \frac{\mathrm{d} r}{\mathrm{~d} R} \frac{\mathrm{d} \omega}{\mathrm{d} R}=\frac{H_{2}}{r} \\
\sigma_{z \theta}=2 W_{l 1}\left[r \frac{\mathrm{d} \chi}{\mathrm{d} R} \frac{\mathrm{d} \omega}{\mathrm{d} R}+\frac{a r k_{\theta}}{R^{2}}+r \psi k_{z}\right] \\
\sigma_{z z}=-p+2 W_{l 1}\left[\left(\frac{\mathrm{d} \omega}{\mathrm{d} R}\right)^{2}+\frac{k_{\theta}^{2}}{R^{2}}+k_{z}^{2}\right] \\
\sigma_{\theta \theta}=-p+2 W_{l 1}\left[\left(r \frac{\mathrm{d} \chi}{\mathrm{d} R}\right)^{2}+\left(\frac{\alpha r}{R}\right)^{2}+(r \psi)^{2}\right] \\
\sigma_{r r}=-p+2 W_{l 1}\left(\frac{\mathrm{d} r}{\mathrm{~d} R}\right)^{2}=-p+2 W_{l 1}\left(\frac{R}{K r}\right)^{2}
\end{gathered}
$$

In the applications that follow, we shall neglect $\mathrm{d} \chi / \mathrm{d} R$ and $\mathrm{d} \omega / \mathrm{d} R$ that are relatively small. This simplifies the numerical procedure of calculation used in what follows.

\section{MATHEMATICAL PROCEDURE OF CALCULATION}

The numerical procedure used in what follows avoids an explicit knowledge of the pseudo-strain energy function $W\left(I_{1}, \lambda_{f}\right)$. If the values of the fiber tension $T$ are known, then we can calculate directly $D_{r}, \sigma_{z \theta}$ and $\sigma_{z z}$ from eqns (4a), (17) and (18). We then proceed by eliminating $-p$ and $W_{I 1}$ from eqns (42-45) and we get

$$
\frac{\sigma_{z \theta}}{\sigma_{z z}-\sigma_{r r}}=\frac{\frac{\left(k_{\theta} a r\right)}{R^{2}}+k_{z} \psi r}{\frac{k_{\theta}^{2}}{R^{2}}+k_{z}^{2}-\frac{R^{2}}{(K r)^{2}}}
$$


and

$$
\frac{\sigma_{z \theta}}{\sigma_{\theta \theta}-\sigma_{r r}}=\frac{\frac{\left(k_{\theta} a r\right)}{R^{2}}+k_{z} \psi r}{\frac{(a r)^{2}}{R^{2}}+(\psi r)^{2}-\frac{R^{2}}{(K r)^{2}}}
$$

Equations (46) and (47) are the basis of the calculation outlined in what follows. We notice for instance from Fig. 5 that $\sigma_{z \theta}$ is zero for a value of $r$ we indicate by $r_{z}$, the corresponding value $R_{z}$ is calculated from eqn (27). The numerator of eqn (46) gives

$$
\alpha k_{\theta}+\psi k_{z} R_{z}^{2}=0
$$

and we have from the incompressibility condition

$$
a k_{z}-\psi k_{\theta}=K
$$

where $K=\left(R_{o}^{2}-R_{i}^{2}\right) /\left(r_{o}^{2}-r_{i}^{2}\right)$ from eqn (27). By solving eqns (48) and (49) we get

$$
\begin{gathered}
a=\frac{k_{z} K R_{z}^{2}}{k_{\theta}^{2}+k_{z}^{2} R_{z}^{2}} \\
\psi=-\frac{k_{\theta} K}{k_{\theta}^{2}+k_{z}^{2} R_{z}^{2}}
\end{gathered}
$$

These two values are then substituted in eqn (46) evaluated at $r_{i}$ and $r_{o}$ with the boundary conditions given by eqn (15). We get the two following relations:

$$
\begin{aligned}
& f\left(k_{z}, k_{\theta}\right)=c f_{i} *\left[k_{\theta}^{2}+R_{i}^{2}\left(k_{z}^{2}-\left(\frac{R_{i}}{K r_{i}}\right)^{2}\right)\right] * \operatorname{det}+k_{\theta} k_{z} K\left(R_{i}^{2}-R_{z}^{2}\right)=0 \\
& g\left(k_{z}, k_{\theta}\right)=c f_{o} *\left[k_{\theta}^{2}+R_{o}^{2}\left(k_{z}^{2}-\left(\frac{R_{o}}{K r_{o}}\right)^{2}\right)\right] * \operatorname{det}+k_{\theta} k_{z} K\left(R_{o}^{2}-R_{z}^{2}\right)=0
\end{aligned}
$$

where $c f_{i}=\sigma_{\mathrm{z} \theta}\left(r_{i}\right) /\left(\sigma_{\mathrm{zz}}\left(r_{i}\right)+P_{i}\right)$ evaluated at $r_{i}, c f_{o}=\sigma_{\mathrm{z} \theta}\left(r_{o}\right) /\left(\sigma_{\mathrm{zz}}\left(r_{o}\right)+P_{o}\right)$ evaluated at $r_{o}$ and $\operatorname{det}=k_{\theta}^{2}+k_{z}^{2} R_{z}^{2}$. The two roots $k_{\theta}$ and $k_{z}$ of eqns (52) and (53) are calculated by using Newton iteration procedure. For this purpose we calculate the Jacobian

$$
J=\left[\begin{array}{ll}
\frac{\partial f}{\partial k_{z}} & \frac{\partial f}{\partial k_{\theta}} \\
\frac{\partial g}{\partial k_{z}} & \frac{\partial g}{\partial k_{\theta}}
\end{array}\right]
$$

We start with an approximation of $k_{z}$ and $k_{\theta}$. The iteration process is expressed in the form

$$
\left[\begin{array}{l}
k_{z} \\
k_{\theta}
\end{array}\right]_{j+1}=\left[\begin{array}{l}
k_{z} \\
k_{\theta}
\end{array}\right]_{j}-J^{-1}\left[\begin{array}{l}
f\left(k_{z}, k_{\theta}\right) \\
g\left(k_{z}, k_{\theta}\right)
\end{array}\right]_{j}
$$


which gives the values of $k_{z}$ and $k_{\theta}$ at the $(j+1)$ th iteration in terms of the preceding $j$ th iteration. Having calculated these two parameters we can then calculate $\alpha$ and $\psi . \sigma_{r r}$ is then calculated from eqn (46) and $\sigma_{\theta \theta}$ from eqn (47).

\section{NUMERICAL APPLICATIONS}

The three simulations described in what follows follow the same steps of calculation. Simulation \#1 is based on data for the fiber tension $T$ calculated from Fig. 7A of Feit [17], and simulations \#2 and \#3 are based on data of $T / P_{i}$ calculated from Fig. 5 of Chadwick [13] who uses in his simulation the same dimension $r_{o}$ and $r_{i}$ reproduced from Feit. Consequently the three simulations use the same values for the outer radius $r_{o}=3.38 \mathrm{~cm}$, the inner radius $r_{i}=1.02 \mathrm{~cm}$ and length $l=3.06 \mathrm{~cm}$.

\subsection{Simulation \#1}

A linear variation of $\gamma(r)$ is assumed (see eqn (33)). The radial contraction force $D_{c}=-D_{r}$ is positive during the systolic phase or late diastolic phase and it is calculated from eqn (4a), both $T$ and $D_{c}$ are
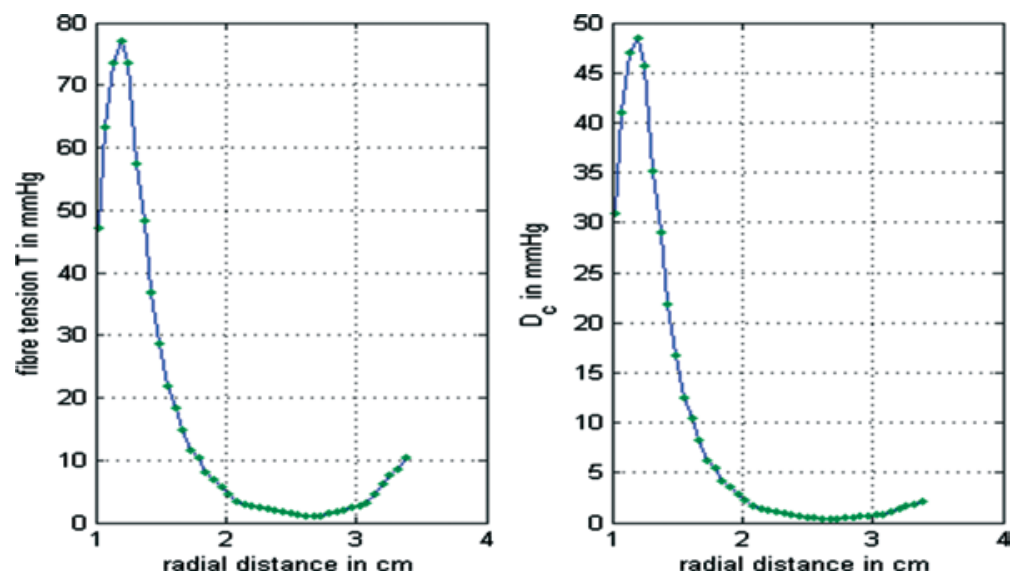

Figure 4: Simulation \#1, radial variation from endocardium to epicardium of the fiber tension $T$ (left), and of the radial active force/unit volume of the myocardium $D_{c}=-D_{r}$ (right).
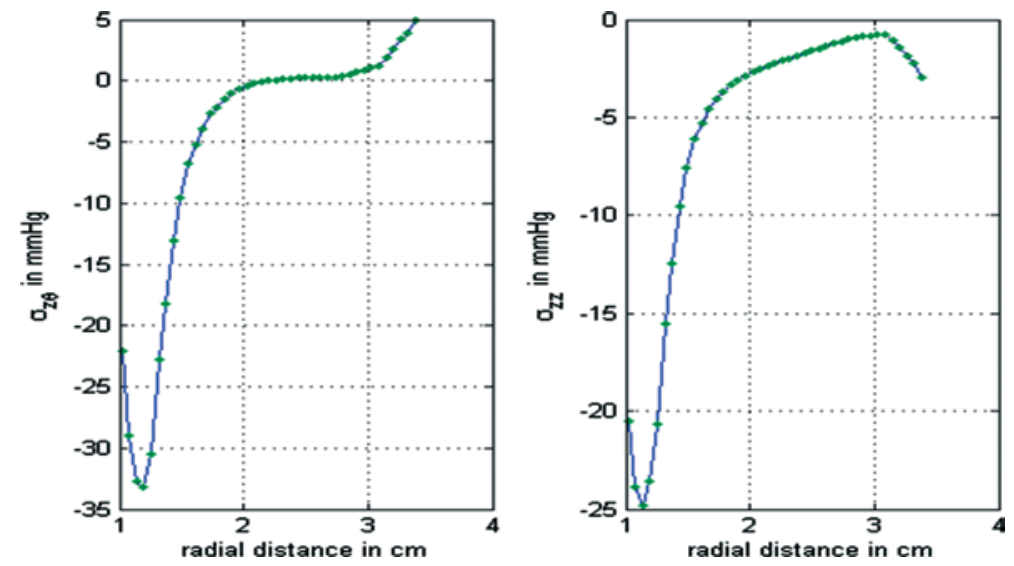

Figure 5: Simulation \#1, radial variation from endocardium to epicardium of the stress component $\sigma_{z \theta}$ (left), and of the axial stress $\sigma_{z z}$ (right). 
plotted against $r$ in Fig. 4. The variation of $\sigma_{z \theta}$ (see eqn (17)) and $\sigma_{z z}$ (see eqn (18)) with $r$ is shown in Fig. 5, $\sigma_{z \theta}=0$ for $r_{z}=2.2 \mathrm{~cm}$ which is used to calculate $R_{z}$ in eqn (48). We have taken in our calculation the inner pressure $P_{i}=10 \mathrm{mmHg}$ from the work of Feit [17] and we have assumed that the outer pressure $P_{o} \approx 0.05 P_{i}$. The corresponding dimensions in the stress free configuration were estimated as $R_{o}=$ $3.48 \mathrm{~cm}$ and $R_{i}=1.15 \mathrm{~cm}, \sigma_{r r}=t_{r r}\left(q_{r r}=0\right)$ and $\sigma_{\theta \theta}$ are calculated as indicated in Section 6 and the results are shown in Fig. 6 together with $q_{\theta \theta}$ and $t_{\theta \theta}=q_{\theta \theta}+\sigma_{\theta \theta}$. Calculated values of $k_{\theta}=-0.4699, k_{z}=0.9350$, $\alpha=1.0603$ and $\psi=0.1011$. Finally the variation with respect to $r$ of $W_{\lambda n}$ and $W_{I 1}$ is shown in Fig. 7 .

\subsection{Simulation \#2}

A linear variation of $\gamma(r)$ is assumed (see eqn (33)). The radial contraction force $D_{c}=-D_{r}$ is positive during the systolic phase or late diastolic phase and it is calculated from eqn (4a), both $T / P_{i}$ and $D_{c}$ are plotted against $r$ in Fig. 8. The variation with respect to $r$ of $\sigma_{z \theta}$ (see eqn (17)) and $\sigma_{z z}$ (see eqn (18)) is shown in Fig. 9, $\sigma_{z \theta}=0$ for $r_{z}=2.2 \mathrm{~cm}$ which is used to calculate $R_{z}$ in eqn (48). We have
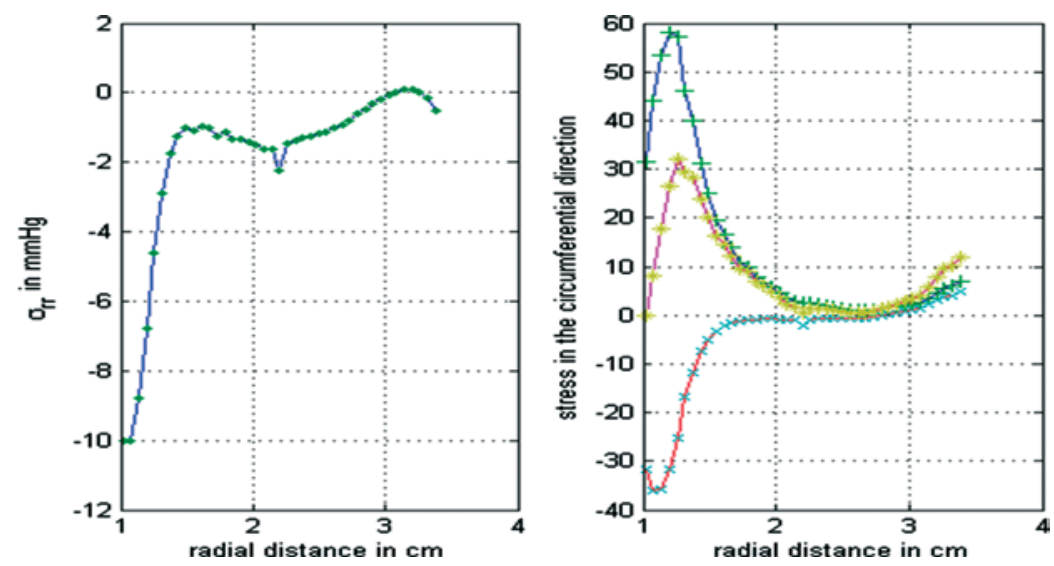

Figure 6. Simulation \#1, radial variation from endocardium to epicardium of the radial stress $\sigma_{r r}$ (left), and of the circumferential stress $q_{\theta \theta}(+), \sigma_{\theta \theta}(\mathrm{x})$ and (*) $t_{\theta \theta}=q_{\theta \theta}+\sigma_{\theta \theta}$ (right).
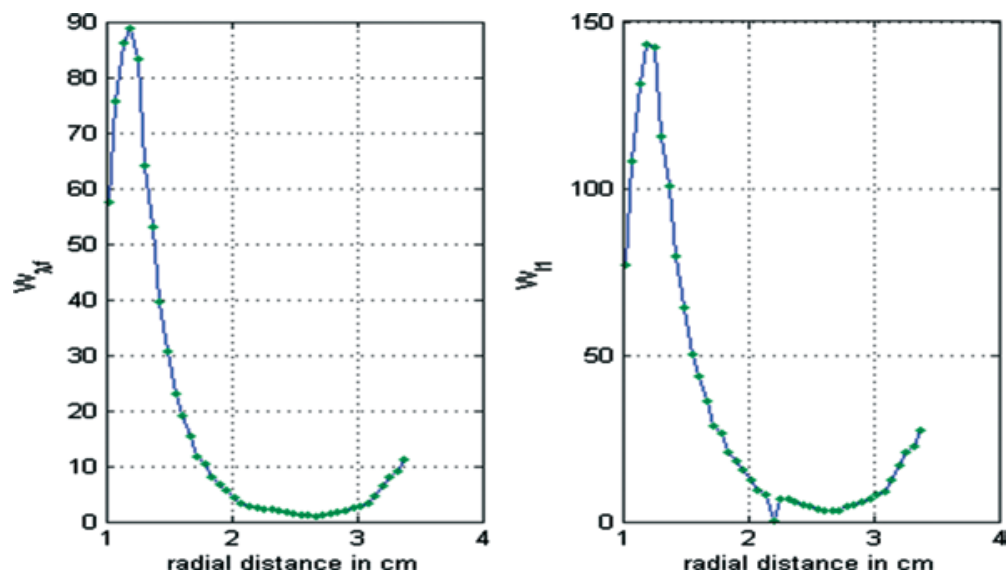

Figure 7: Simulation \#1, radial variation from endocardium to epicardium of $W_{\lambda f}$ (eqn (39)) (left), and $W_{I 1}$ (eqn (42)) (right). 
taken in our calculation the inner pressure $P_{i}=1 \mathrm{mmHg}$ based on the work of Chadwick [13] and we have assumed that the outer pressure $P_{o} \approx 0$. The corresponding dimensions in the stress free configuration were estimated as $R_{o}=3.455 \mathrm{~cm}$ and $R_{i}=1.08 \mathrm{~cm}, \sigma_{r r}=t_{r r}\left(q_{r r}=0\right)$ and $\sigma_{\theta \theta}$ are calculated as indicated in Section 6 and the results are shown in Fig. 10 together with $q_{\theta \theta}$ and $t_{\theta \theta}=q_{\theta \theta}+\sigma_{\theta \theta}$. Calculated values of $k_{\theta}=0.2011, k_{z}=0.9905, \alpha=1.0388$ and $\psi=-0.0413$. Finally the variation with respect to $r$ of $W_{\lambda n}$ and $W_{I 1}$ is shown in Fig. 11.

\subsection{Simulation \#3}

A cubic variation of $\gamma(r)$ is assumed (see eqn (34)). The radial contraction force $D_{c}=-D_{r}$ is positive during the systolic phase or late diastolic phase and it is calculated from eqn (4a), both $T / P_{i}$ and $D_{c}$ are plotted against $r$ in Fig. 12. The variation with respect to $r$ of $\sigma_{z \theta}$ (see eqn (17)) and $\sigma_{z z}$ (see eqn (18)) is shown in Fig. 13, $\sigma_{z \theta}=0$ for $r_{z}=2.2 \mathrm{~cm}$ which is used to calculate
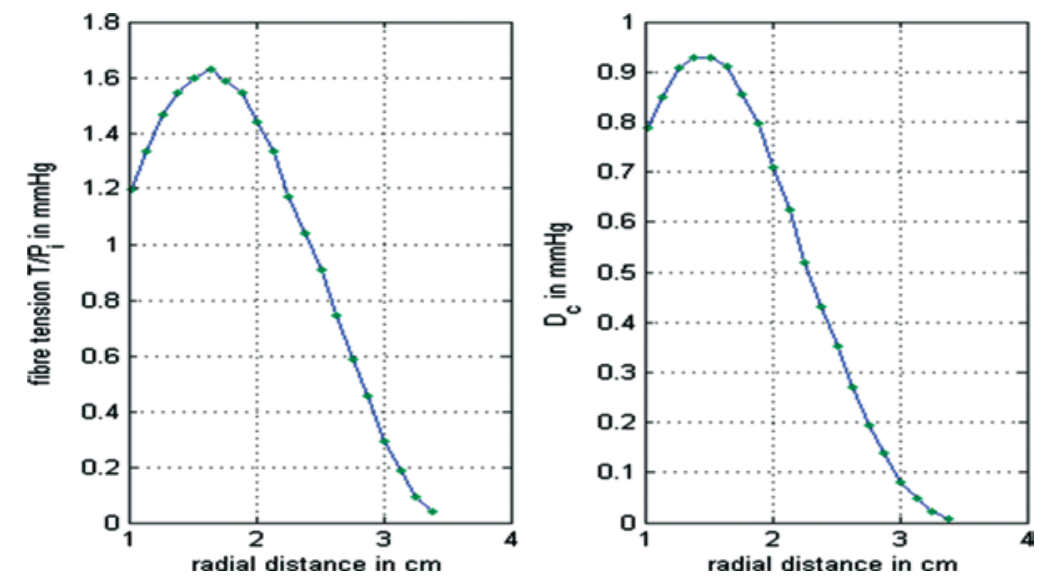

Figure 8: Simulation \#2, radial variation from endocardium to epicardium of the fiber tension $T / P_{i}$ (left), and of the radial active force/unit volume of the myocardium $D_{c}=-D_{r}$ (right).
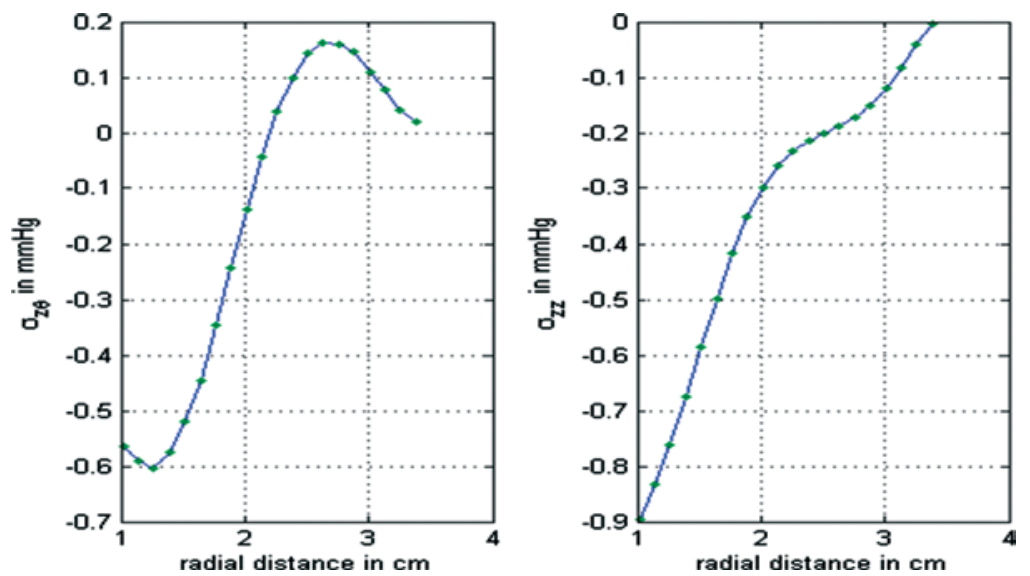

Figure 9: Simulation \#2, radial variation from endocardium to epicardium of the stress component $\sigma_{z \theta}\left(\right.$ left), and of the axial stress $\sigma_{z z}$ (right). 

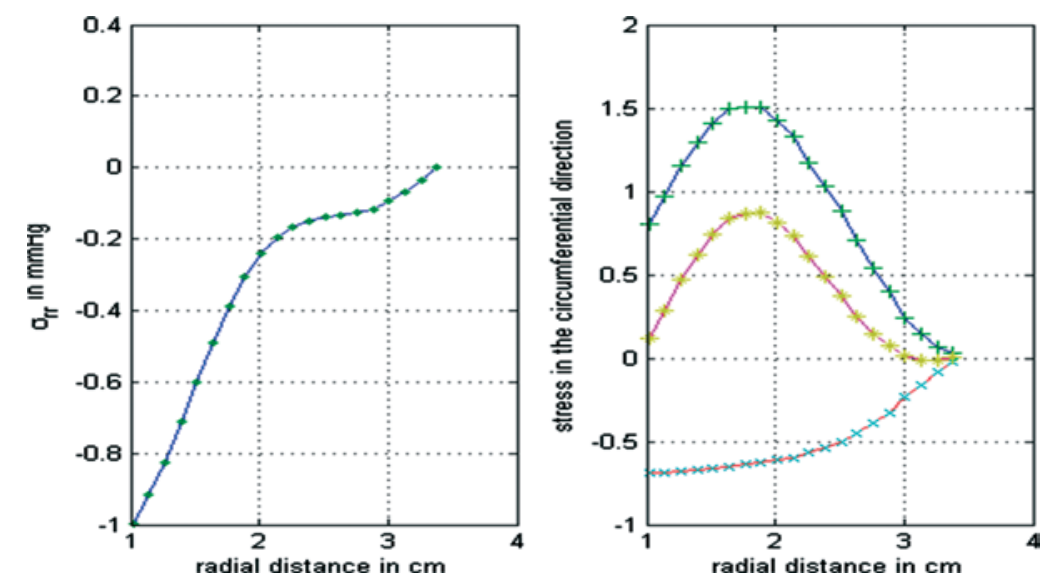

Figure 10: Simulation \#2, radial variation from endocardium to epicardium of the radial stress $\sigma_{r r}$ (left), and of the circumferential stress $q_{\theta \theta}(+), \sigma_{\theta \theta}(\mathrm{x})$ and $(*) t_{\theta \theta}=q_{\theta \theta}+\sigma_{\theta \theta}$ (right).
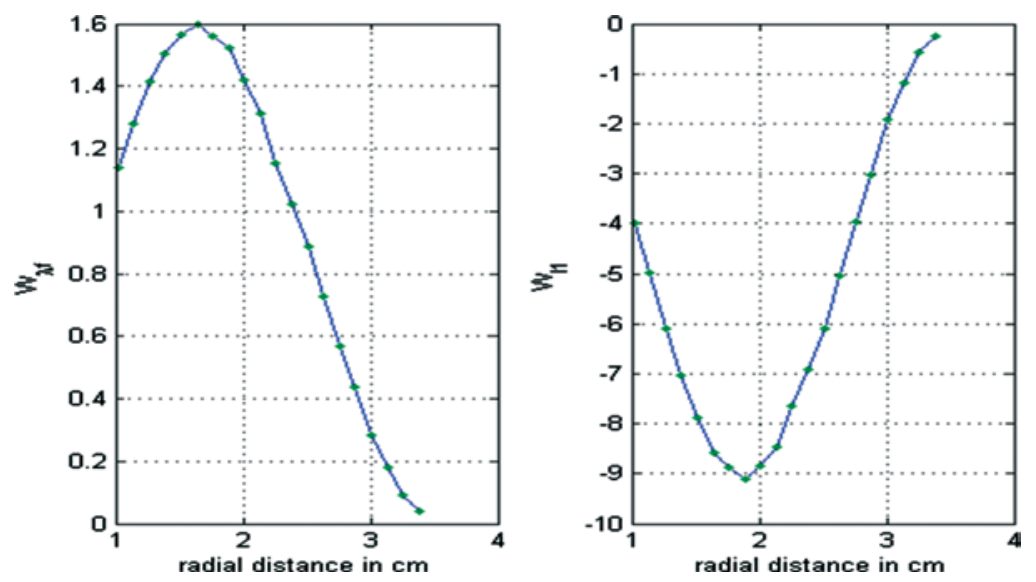

Figure 11: Simulation \#2, radial variation from endocardium to epicardium of $W_{\lambda f}$ (eqn (39)) (left), and $W_{I 1}$ (eqn (42)) (right).

$R_{z}$ in eqn (48). We have taken in our calculation the inner pressure $P_{i}=1 \mathrm{mmHg}$ based on the work of Chadwick [13] and we have assumed that the outer pressure $P_{o} \approx 0$. The corresponding dimensions in the stress free configuration were estimated as $R_{o}=3.455 \mathrm{~cm}$ and $R_{i}=1.08 \mathrm{~cm}$, $\sigma_{r r}=t_{r r}\left(q_{r r}=0\right)$ and $\sigma_{\theta \theta}$ are calculated as indicated in Section 6 and the results are shown in Fig. 14 together with $q_{\theta \theta}$ and $t_{\theta \theta}=q_{\theta \theta}+\sigma_{\theta \theta}$. Calculated values of $k_{\theta}=0.2011, k_{z}=0.9905$, $\alpha=1.0388$ and $\psi=-0.0413$. Finally the variation with respect to $r$ of $W_{\lambda n}$ and $W_{I 1}$ is shown in Fig. 15.

The three examples show the wide variation of $W_{I 1}$ from one example to another. The values of $R_{o}$ and $R_{i}$ chosen are not unique, the simulation is very sensitive to the values of the parameters chosen and there is unfortunately no systematic and simultaneous measurement of all the parameters needed for our simulation. On the other hand one should notice the similarity of 

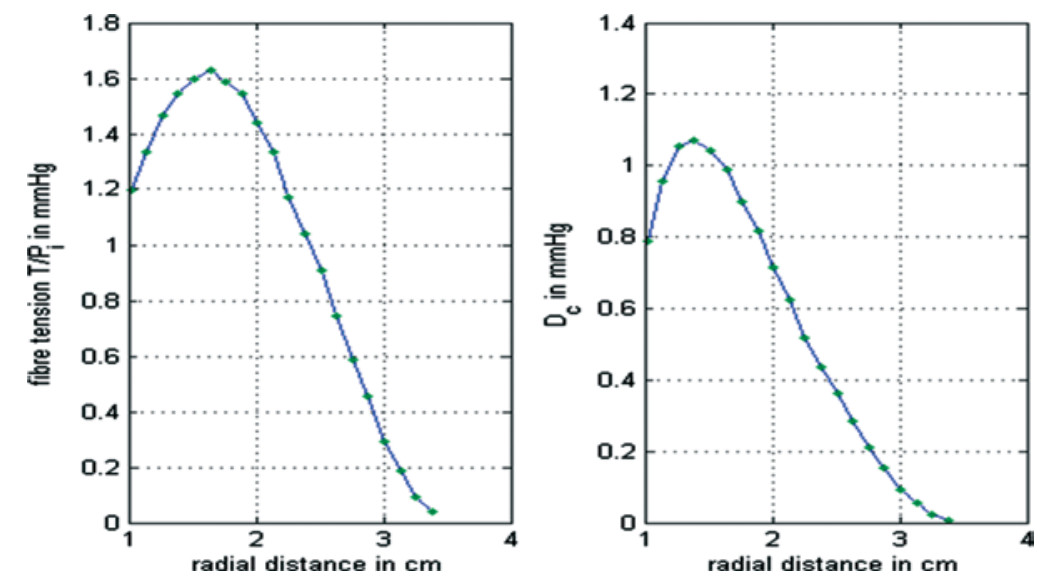

Figure 12: Simulation \#3, radial variation from endocardium to epicardium of the fiber tension $T / P_{i}$ (left), and of the radial active force/unit volume of the myocardium $D_{c}=-D_{r}$ (right).
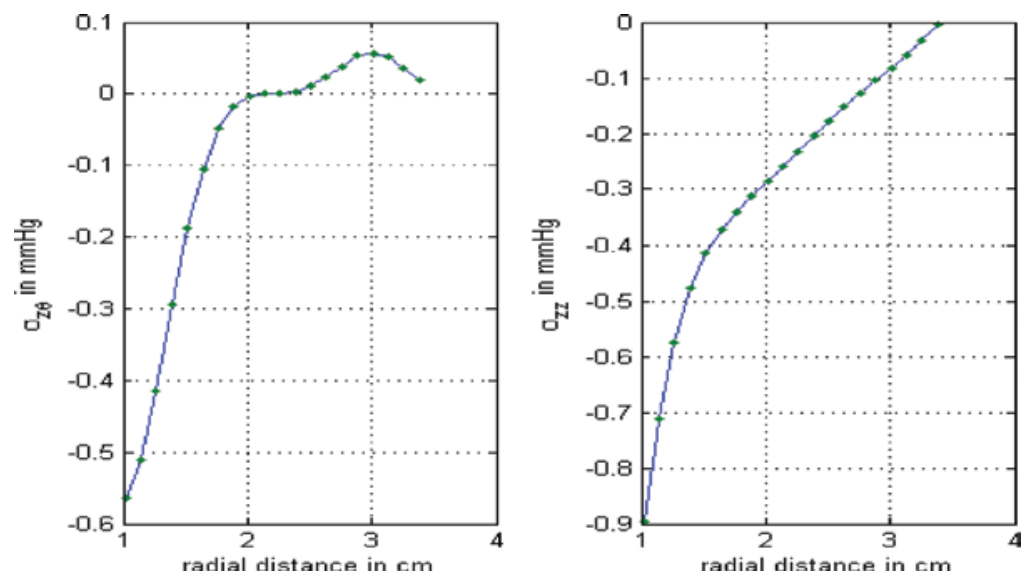

Figure 13: Simulation \#3, radial variation from endocardium to epicardium of the stress component $\sigma_{z \theta}$ (left), and of the axial stress $\sigma_{z z}$ (right).

the curves for $W_{\lambda n}$ and $T$ (or $T / P_{i}$ ), which is due to the fact that the stretch ratio $\lambda_{n}$ is varying around unity (see eqn (39)). The calculation of the components of the stress has been carried out without explicit knowledge of the pseudo-strain energy function $W\left(I_{1}, \lambda_{f}\right)$. The next step is to study a procedure by which one can recover $W\left(I_{1}, \lambda_{f}\right)$ from the knowledge of the partial derivatives $W_{\lambda f}$ and $W_{I 1}$.

\section{CONCLUSION}

The total stress in the myocardium can be split into two components, a component due to the deformation of the passive isotropic medium of the myocardium and a component induced by the active muscular fibers $T$. By using this approach we have been able under some assumptions to derive these 

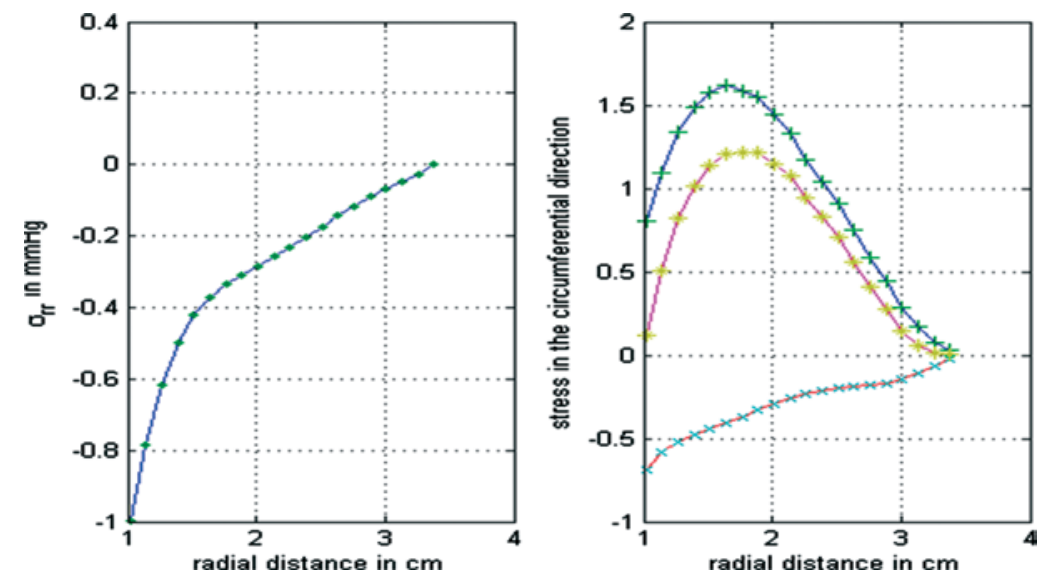

Figure 14: Simulation \#3, radial variation from endocardium to epicardium of the radial stress $\sigma_{r r}$ (left), and of the circumferential stress $q_{\theta \theta}(+), \sigma_{\theta \theta}(\mathrm{x})$ and $(*) t_{\theta \theta}=q_{\theta \theta}+\sigma_{\theta \theta}$ (right).
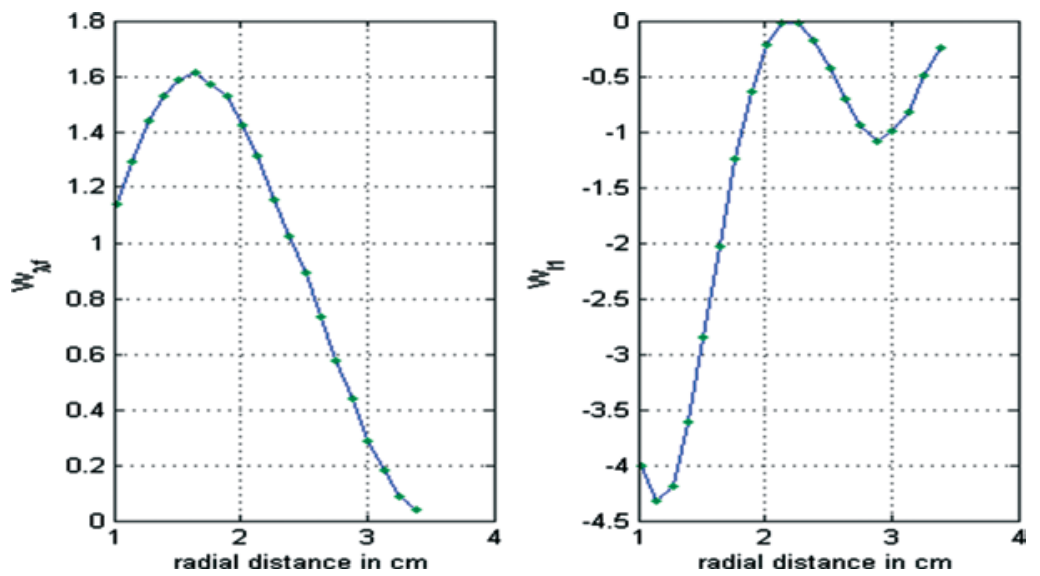

Figure 15: Simulation \#3, radial variation from endocardium to epicardium of $W_{\lambda f}$ (eqn (39)) (left), and $W_{I 1}$ (eqn (42)) (right).

parts of the stress without explicit knowledge of the pseudo-strain energy function $W$. This method is sensitive to the parameters chosen in the calculation, unfortunately not all are measured simultaneously. By integrating the partial derivatives of $W$ one can possibly gain better understanding of how one can choose directly a mathematical model for $W$.

\section{REFERENCES}

[1] Humphrey, J.D. \& Yin, F.C.P., Constitutive relations and finite deformations of passive cardiac tissue II: stress analysis in the left ventricle. Circulation Research, 65, pp. 805-817, 1989.

[2] Guccione, J.M., McCulloch, A.D. \& Waldman, L.K., Passive material properties of intact ventricular myocardium determined from a cylindrical model. Journal of Biomechanical Engineering, 113, pp.42-55, 1991. Comment by Chaudhry, H.R., Journal of Biomechanical Engineering, 118, pp. 262-263, 1996. doi:10.1115/1.2795972, doi:10.1115/1.2795971 
[3] Yang, M., Taber, L.A. \& Clark, E.B., A nonlinear poroelastic model for the trabecular embryonic heart. Journal of Biomechanical Engineering, 116, pp. 213-223, 1994. doi:10.1115/1.2895722

[4] Demiray, H., Stresses in ventricular wall. Journal of Applied Mechanics, 43 , pp. 194-197, 1976.

[5] Shoucri, R.M., Equivalence of two approaches to study the stress-strain relation in the myocardium. Modelling in Medicine and Biology VIII, eds C.A. Brebbia, WIT Press: Southampton \& Boston, pp. 3-16, 2009. doi:10.1109/51.677175

[6] Shoucri, R.M., Active and passive stresses in the myocardium. American Journal of Physiology, 279, pp. H2519-H2528, 2000.

[7] Shoucri, R.M., Studying the mechanics of left ventricular contraction. IEEE Engineering in Medicine and Biology Magazine, 17, pp. 95-101, 1998.

[8] Shoucri, R.M., Theoretical study of pressure-volume relation in left ventricle. American Journal of Physiology, 260, pp. H282-H291, 1991.

[9] Shoucri, R.M., The pressure-volume relation and the mechanics of left ventricular contraction. Japanese Heart Journal, 31, pp.713-729, 1990.

[10] Spencer, A.J.M., Deformation of FIber-reinforced Materials, Clarendon Press: Oxford, UK, 1972.

[11] Holzapfel, G.A., Gasser, T.C. \& Ogden, R.W., Comparison of a multi-layer structural model for arterial walls with a Fung-type model, and issues of material stability. Journal of Biomechanical Engineering, 126, pp. 264-274, 2004. doi:10.1115/1.1695572

[12] Peskin, C.S., Mathematical aspects of heart physiology, New York University, Courant Institute of Mathematical Sciences, 1975.

[13] Chadwick, R.S., The myocardium as a fluid-fiber continuum: passive equilibrium configurations. Advances in Bioengineering ed D.C. Viano, The American Society of Mechanical Engineers, pp. 135-138, 1981.

[14] Nevo, E. \& Lanir, Y., Structural finite deformation model of the left ventricle during diastole and systole. Journal of Biomechanical Engineering, 111, pp. 342-349, 1989. doi: $10.1115 / 1.3168389$

[15] Arts, T., Bovendeerd, P.H.M., Prinzen, F.W. \& Reneman, R.S., Relation between left ventricular cavity.pressure and volume and systolic fiber stress and strain in the wall. Biophysical Journal, 59, pp.93-102, 1991. doi:10.1016/S0006-3495(91)82201-9

[16] Tözeren, A., Static analysis of the left ventricle. Journal of Biomechanical Engineering, 105, pp. 39-46, 1983. doi:10.1115/1.3138382

[17] Feit, T.S., Diastolic pressure-volume relations and distribution of pressure and fiber extension across the wall of a model left ventricle. Biophysical Journal, 28, pp. 143-166, 1979. doi:10.1016/S0006-3495(79)85165-6 